\title{
Lack of evidence about the effectiveness of the different denture cleaning methods
}

\author{
Abstracted from \\ de Souza RF, de Freitas Oliveira Paranhos H, Lovato da Silva CH, Abu-Naba'a L, Fedorowicz Z, Gurgan CA. \\ Interventions for cleaning dentures in adults. Cochrane Database Syst Rev 2009; issue 4 \\ Address for correspondence: Luisa Fernandez Mauleffinch, Cochrane Oral Health Group, \\ MANDEC, School of Dentis : luisa.fernandez@manchester.ac.uk \\ This paper is based on a Cochrane Review published in The Cochrane Library 2009, Issue 4 \\ (see www.thecochranelibrary.com for information). Cochrane Reviews are regularly updated as new evidence emerges \\ and in response to feedback, and The Cochrane Library should be consulted for the most recent version of the review.
}

\section{Question: What is the safest and most effective method of cleaning removable dentures?}

Data sources Relevant studies were sourced by searching the Cochrane Oral Health Group Trials Register, Cochrane CENTRAL (Cochrane Library), Medline, Embase, LILACS and CINAHL. There were no language restrictions.

Study selection Randomised controlled trials (RCT) were considered if they compared any mechanical method (eg, brushing or ultrasound) or chemical (eg, enzymes, sodium hypochlorite, oral rinses or peroxide solutions) in adults over the age of 18 years who wore removable partial dentures or complete dentures.

Data extraction and synthesis Two independent review authors screened and extracted information from, and independently assessed the risk of bias in, the included trials.

Results Although six RCT were included in this review, the wide range of different interventions and outcome variables did not permit pooling of data in a meta-analysis. Isolated reports indicated that chemicals and brushing appear to be more effective than placebo in reducing plaque coverage and reducing microbial counts of anaerobes and aerobes on complete denture bases.

Conclusions There is a lack of evidence about the comparative effectiveness of the different denture cleaning methods considered in this review. Few well-designed RCT were found. Future research should focus on comparisons between mechanical and chemical methods, should assess the association between methods, and consider primary variables and costs.

\section{Commentary}

Dentures are cleaned to prevent malodour and to remove stain, plaque and calculus from the denture surfaces. There are a large number of solutions, pastes and powders available for cleaning dentures, with a variety of claims for their relative efficacies. Previous reviews of studies of the efficacy of denture cleansers have indicated that the results obtained are dependent on the methods used to evaluate the efficacy. ${ }^{1,2}$ In-vitro assays may overestimate efficacy. The difficulties facing investigators have been compounded by recent findings showing that the efficacy of a given method is also dependent on the type of microbial biofilm. ${ }^{3}$

The present review uses rigorous Cochrane methodology that considers only RCT. Unfortunately, only six studies were identified as being suitable for inclusion and all studies were of edentulous subjects. The wide range of interventions and outcomes of those studies inevitably limits the practical usefulness of the findings. The choice of the health of particular denture-bearing areas (soft tissues, periodontal tissues and teeth) and participants' satisfaction and preference as primary outcomes is open to discussion. The health of the tissues is an indirect outcome and patient preference is more a predictor of use, and therefore of effectiveness, rather than efficacy. The outcomes of the studies included in the review were all classed as secondary outcome measures: denture plaque coverage area, plaque score (three studies) and microbial counts from samples of specific areas of dentures (four studies). Nevertheless, the conclusions are robust and serve to emphasise our current lack of scientific knowledge of the relative efficacy of denture cleansers. An inference from the findings that chemical methods and brushing were more effective than placebo or no cleaning is that some cleaning is generally better than no cleaning. Comparisons of efficacy are of interest and importance, but surveys show that a large number of people do not know how to clean their dentures satisfactorily and suggest that dentists may fail to educate patients (or carers) in even basic dental hygiene procedures. ${ }^{4}$

\section{Robert Jagger}

Division of Restorative Dentistry, Department of Oral and Dental Science, University of Bristol, Bristol, UK

1. Nikawa $\mathrm{H}, \mathrm{Hamada} T$, Yamashiro $\mathrm{H}, \mathrm{Kumagai} \mathrm{H}$. A review of in vitro and in vivo methods to evaluate the efficacy of denture cleansers. Int J Prosthodont 1999; 12: 153-159.

Abelson DC. Denture plaque and denture cleansers: review of the literature. Gerodontics 1985; 1: 202-206.

3. Paranhos HF, Silva-Lovato $\mathrm{CH}$, de Souza RF, et al. Effect of three methods for cleaning dentures on biofilms formed in vitro on acrylic resin. J Prosthodont 2009; 18: 427-431. 4. Jagger DC, Harrison A. Denture cleansing - the best approach. Br Dent J 1995; 178:
$413-417$.

Evidence-Based Dentistry (2009) 10, 109. doi:10.1038/sj.ebd.6400684 